\title{
Optimal Distribution of N-Team Interacting Decision Makers with Hierarchical Command Inputs That Are Predicated on Order Statistics
}

\author{
Michael Olatunji Oladejo, Uchenwa Linus Okafor, Darius Tienhua Chinyio, \\ Celestine Ozoemena Uwa \\ Nigerian Defence Academy, Kaduna, Nigeria \\ Email: mikeoladejo2012@gmail.com, mooladejo@nda.edu.ng, linusokafor@gmail.com, ulokafor@nda.edu.ng, \\ dtchinyio@nda.edu.ng,couwa@nda.edu.ng
}

How to cite this paper: Oladejo, M.O., Okafor, U.L., Chinyio, D.T. and Uwa, C.O. (2019) Optimal Distribution of N-Team Interacting Decision Makers with Hierarchical Command Inputs That Are Predicated on Order Statistics. Open Journal of Optimization, 8, 1-14.

https://doi.org/10.4236/ojop.2019.81001

Received: November 30, 2018

Accepted: February 12, 2019

Published: February 15, 2019

Copyright $\odot 2019$ by author(s) and Scientific Research Publishing Inc. This work is licensed under the Creative Commons Attribution International License (CC BY 4.0).

http://creativecommons.org/licenses/by/4.0/

\begin{abstract}
The spectral analysis of simulated N-team of interacting decision makers with bounded rationality constraints of Oladejo, which assumes triangular probability density function of command inputs is hereby restructured and analysed, to have hierarchical command inputs that are predicated on order statistics distributions. The results give optimal distributions.

\section{Keywords}

Decision Maker, Command and Control, Bounded Rationality Constraints, Hierarchical, Conditional Probability Density Function, Situation Report, Computational Intelligence and Information Management
\end{abstract}

\section{Introduction}

The Double-Team of decision Maker Model that Boettcher and Levis developed [1] was extended and generalized by Oladejo [2] to N-Teams of Decision Makers with $\mathrm{N}^{\mathrm{n}}$ Bounded Rationality Constraints. A Spectral Analysis of the model was provided by Oladejo [3], where command inputs were based on uniform distribution. This work considered the same model with modified command inputs that were hierarchical and whose distribution was predicated on order statistics. In this new work, the command inputs were categorized according to the superiority of the commander who was next higher in rank to the officer making input. The analytical procedure of optimizing convoluted strategies was used to derive the optimal distribution functions of the hierarchical command inputs. In the previous work there was a mixture of vertical and horizontal signal commu- 
nications, whereas in this recent research consideration given to all signal communications must be ascending serially and vertically. This procedure is endemic with optimal sequencing [4], optimal choices [5], optimization of vending outlets [4] [6] [7], optimal transportation security [8], optimal maintenance policy [9] and optimized system models [10] [11] [12] [13].

The Model: The model of N-Team interacting decision makers with bounded rationality constraints was decomposed into coherent component strategies which were then used to analyze the system of specified $\mathrm{C}^{3} \mathrm{I}$.

\section{Symbols}

$X^{i}$ is the input signal having exponential tempo/inter arrival times of the $I^{\text {th }}$ team.

$\pi$ is the portioning algorithm of inputs to respective DM.

$u$ is the internal decision.

$f$ is the algorithm for process $u^{i}$ to obtain the battle scenario.

$q^{i}$ is the $i^{\text {th }}$ team input regulator.

$Z^{i}$ is the initial situational assessment.

$\bar{z}^{i}$ is the final situational assessment.

$g_{C}^{A^{i}}, g_{C}^{B^{i}}$ are the internal coordination strategies using SITREP $z^{\prime}$ s and $v^{\dot{ }}$, the command input to the $i^{\text {th }}$ team.

$Z^{\prime}$ is situation assessment from other organizational members (SITREP or Feedback).

$v$ is the final choice of decision.

$V^{\prime}$ is the command input.

$\bar{v}^{i}$ is the output of DM $i$.

$\bar{v}$ is the final choice or response.

$Y$ is the output or desired result.

$h_{j}$ is the processing algorithm for the final choice leading to $Y$.

$h^{i}$ is the algorithm for process $v^{j}$.

$P(v \mid z)$ is response selection strategy that maps $z$ to $Y$ in the absence of $v^{\prime}$ and determines choices of $h_{j}$.

$P\left(\bar{v} \mid \bar{z}, v^{\prime}\right)$ is response selection strategy that maps $z$ and $V^{\prime}$ to $V$, and it also determines choices of $h_{j}$.

$H(x)=-\sum_{x} p(x) \log _{2} p(x)$ is entropy of inputs, where $p(x)$ is probability or uncertainty associated with $N$ random variables, $X$.

$T\left(x, z^{\prime}, v^{\prime}, Y\right)$ is mutual information or transmission or throughput between inputs $x, Z^{\prime}, V^{\prime}$ and output $Y$.

$G_{t}$ is the throughput.

$G_{b}$ is the blockage.

$G_{n}$ is the noise.

$G_{c}$ is the internal coordination.

$\mathbb{H}$ is the entropy of a random variable with probability $p$ for taking one or two values, which also gives the amount of information transmitted.

$A$ is the set of situational assessment options or strategies. 
$B$ is the set of available choices of tactics, modus operandi to remedy problem. $\psi$ is the convolution of strategies which is the assumed combined effects of process activities.

where

$$
\begin{aligned}
& j_{11}=1,2, \cdots, U^{1}, j_{21}=1,2, \cdots, V^{1}, j_{12}=1,2, \cdots, U^{2}, j_{22}=1,2, \cdots, V^{2}, \cdots, \\
& j_{1 N}=1,2, \cdots, U^{N}, \cdots, j_{2 N}=1,2, \cdots, V^{N}
\end{aligned}
$$

This is the structure of the generalized developed model as shown below:

The generalized developed model of Figure 1 is as shown:

$$
\begin{gathered}
\frac{\operatorname{DMt}(t=1,2, \cdots, N)}{G^{t}=T\left(X^{t}, Z^{t(N-1)}, \cdots, Z^{t 1} ; V^{(N-1) 1} Z^{1 t} ; \cdots, Z^{(N-1) t} ; Y^{t}\right)+H\left(X^{t} ; Z^{t(N-1)} ; Z^{t 1} ; V^{(N-1)^{\prime}}\right)} \\
-G_{t}^{t}+H\left(u^{t}\right)+H_{z}\left(v^{t}\right)+\sum_{i=1}^{U^{t}}\left[p_{i} g_{c}^{i}\left(p X^{t}\right)+\alpha_{i} \mathbb{H}\left(p_{i}\right)\right]+H\left(Z^{t}, Z^{1 t}, \cdots, Z^{(N-1) t}\right) \\
+g_{c}^{A^{t}}\left(p\left(Z^{t}\right), p\left(Z^{t 1}\right), \cdots, p\left(Z^{t(N-1)}\right)\right)+g_{c}^{B^{t}}\left(p\left(Z^{t}\right), p\left(V^{(N-1)^{\prime}}\right)\right) \\
+\sum_{j=1}^{V^{t}}\left[p_{j} g_{c}^{j}\left(p\left(Z^{t} / V^{t}\right)\right)+\alpha_{j} \mathbb{H}\left(p_{j}\right)\right]+H\left(Y^{t}\right)+H\left(Z^{t}\right)+H\left(\bar{Z}^{t}\right)+H\left(Z^{t}, V^{t}\right) \\
+T\left(X^{t}: Z^{t 1} ; \cdots, Z^{t(N-1)}\right)+T Z^{-t}\left(X^{t} ; Z^{t 1}, \cdots, Z^{t(N-1)}: V^{(N-1)^{\prime}}\right)
\end{gathered}
$$

\section{Analysis}

Strategies which are probability density functions (pdfs) are given as follows:

$$
U^{i}\left(x^{i}\right) \sim \text { exponential inter arrival times or tempo of operations: }
$$

$\frac{1}{\theta} \mathrm{e}^{-t / \theta}, t, \theta>0$

$f^{i}\left(u^{i}\right) \sim$ normal because of a defined goal (i.e. goal is focus):

$$
\frac{1}{\sqrt{2 \pi \sigma}} \mathrm{e}^{\frac{-1}{2}(x-\mu)^{2} / \sigma^{2}},-\infty<x, \mu<\infty, \sigma^{2}>0
$$

$A\left(z^{i}\right) \sim$ uniform distribution: $\frac{1}{N}$ or $\frac{x}{b-a}, x=0,1,2, \cdots, N$ or $a<x<b$, respectively

$$
v^{\prime} \sim \operatorname{triangular} \frac{\bar{V}}{C}, 0<V C, F=R=\left.\frac{v^{-2}}{2 C}\right|_{0} ^{c}, v^{-1}=2 R,\left(\text { i.e } \text { for } v^{-1}=c\right)
$$

$g_{C}^{k}$ is internal coordination strategy of corresponding algorithm which depends on the distribution of their respective inputs.

$g_{c}^{A^{1}} \sim$ Bernoull: $p^{x} q^{1-x}, x=0,1$ (appropriate strategies are employed. Hence, $\sum X_{i}$ is binomial and all samples are appropriate. These are the internal coordination strategies among situational assessments represented by set $A^{1}$ ).

$g_{c}^{B^{1}} \quad \sim$ geometric: $p q^{x}, x=1,2, \cdots$, depending on $x$ (the strategies would result in a success hence $\sum x_{1}$ is negative binomial that all samples are successful. 


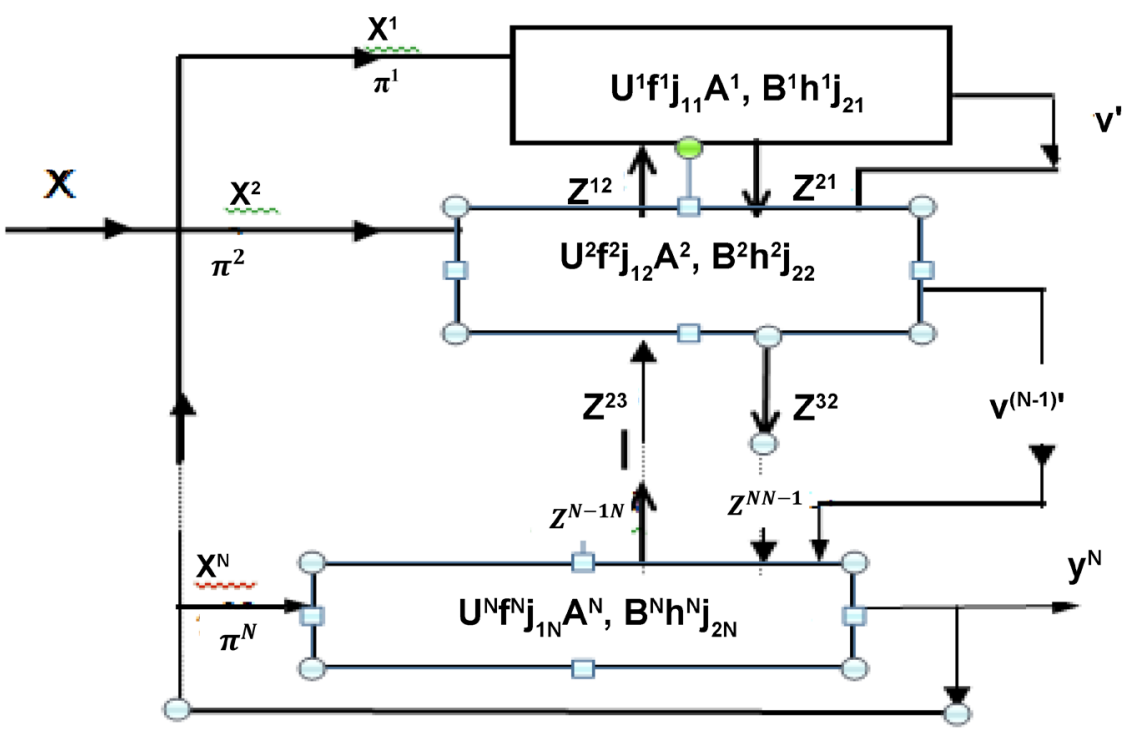

Figure 1. N-Team of interacting decision makers with bounded rationality.

These are the internal coordination strategies among final choices and command inputs represented by set $\left.B^{1}\right)$.

$h\left(v^{\prime}\right) \sim$ Weibull (due to reliability of subsystem before reaching final stage: $\left.\alpha \beta X^{\beta-1} e^{-\alpha} X^{\beta}, X>0, \alpha, \beta>0\right)$.

$\mu(X) \sim \operatorname{expo}$ (this is due to random occurrences).

$F(u) \sim \operatorname{normal}$ (i.e. geared towards a goal).

$A(z) \sim$ uniform (simple random sample, equally likely samples).

$B\left(z, \bar{v} \mid \vec{v}^{\prime}\right) \sim$ conditional jpdf $\{$ (uniform $\times$ triangular $) \times\left(\begin{array}{lll}g_{C}^{A} & g_{C}^{B}\end{array}\right)$ yielding interaction due to $\vec{z}$ s to determine choices of $v$ depending on $\vec{v}^{-1}$ from superior command\}.

$h(\bar{v}) \sim$ Weibull (output $Y$ depends on survivability or reliability of other systems components).

$v^{\prime} \sim$ order Statistic-Hierarchical command inputs.

Now if command inputs $\bar{V}^{-1}$ has pdf Ordered statistics: $f\left(y_{n}\right), f\left(y_{1}\right)$, $f\left(y_{K}\right), f\left(y_{i}, y_{j}\right)$

$$
\begin{aligned}
& g_{n}\left(y_{n}\right)=n\left[F\left(y_{n}\right)\right]^{n-1} f\left(y_{n}\right), a<y_{n}<b \\
& g_{1}\left(y_{1}\right)=n\left[1-F\left(y_{1}\right)\right]^{n-1} f\left(y_{1}\right), a<y_{1}<b
\end{aligned}
$$

Let $g\left(y_{i}\right)=i$ be hierarchical transformations of signal inputs

$$
\begin{aligned}
g_{k}\left(y_{k}\right)= & \frac{n !}{(k-1)(n-k) !}\left[F\left(y_{k}\right)\right]^{k-1}\left[1-F\left(y_{k}\right)\right]^{n-k} f\left(y_{k}\right), a<y_{k}<b \\
g_{i j}\left(y_{i}, y_{j}\right)= & \frac{n !}{(i-1) !(j-i-1) !(n-j) !}\left[F\left(y_{i}\right)\right]^{i-1}\left[F\left(y_{j}\right)-F\left(y_{i}\right)\right]^{j-i-1} \\
& \times\left[1-F\left(y_{j}\right)\right]^{n-j} f\left(y_{i}\right) f\left(y_{j}\right), a<y_{k}<b
\end{aligned}
$$

and zero elsewhere. 


\section{Methodology}

An analytical approach was used to derive the optimal distributions of the convoluted strategies. Since the various events are independent the convolution of strategies was obtained by their product. The derivative of this convoluted strategy was equated to zero then solved.

$B(\bar{z}, \bar{v} \mid \vec{v}) \sim$ discrete conditional jpdfs is given by (uniform $\times$ order statistic)

$\times\left(g_{C}^{A} \cdot g_{C}^{B}\right)$.

$B\left(\bar{z}, \bar{v} \mid \vec{v}^{\prime}\right)$ of first/initial command input.

\subsection{Derivation of pdf}

$$
\begin{gathered}
B\left(\bar{z}, \bar{v} \mid \vec{v}^{\prime}\right)=\frac{1}{N} \cdot N\left[1-F\left(y_{1}\right)\right]^{N-1} f\left(y_{1}\right)\left(\begin{array}{c}
N \\
x
\end{array}\right) P^{x} q^{N-x}\left(\begin{array}{c}
N-1 \\
x-1
\end{array}\right) P^{x} q^{N-x} \\
=\left[1-F\left(y_{1}\right)\right]^{N-1} p\left[\left(\begin{array}{c}
N \\
x
\end{array}\right) P^{x} q^{N-x}\right]^{2}=\left[1-F\left(y_{1}\right)\right]^{N-1} p\left[\left(\begin{array}{l}
N \\
y_{1}
\end{array}\right) P^{y_{1}} q^{N-y_{1}}\right]^{2} \cdot \frac{y_{1}}{N}(10) \\
p \equiv f\left(y_{1}\right), y_{1}=x, 1<y_{1}<N \\
F(y)=\int f(y) \mathrm{d} y \quad \text { or } \\
F(y)=\sum f(y), q=1-p
\end{gathered}
$$

$B\left(\bar{z}, \bar{v} \mid \vec{v}^{\prime}\right)$ of final/last command input.

$$
\begin{aligned}
B\left(\bar{z}, \bar{v} \mid \vec{v}^{\prime}\right) & =\frac{1}{N} \cdot N\left[1-F\left(y_{n}\right)\right]^{N-1} f\left(y_{n}\right)\left(\begin{array}{l}
N \\
y_{n}
\end{array}\right) P^{y_{n}} q^{N-y_{n}}\left(\begin{array}{c}
N-1 \\
y_{n}-1
\end{array}\right) P^{y_{n}} q^{N-y_{n}} \\
& =\left[1-F\left(y_{n}\right)\right]^{N-1} p\left[\left(\begin{array}{l}
N \\
y_{n}
\end{array}\right) P^{y_{n}} q^{N-y_{n}}\right]^{2}=\frac{y_{n}}{N}, p \equiv f\left(y_{n}\right), a<y_{n}<b
\end{aligned}
$$

$B(\bar{z}, \bar{v} \mid \vec{v})$ of intermediate $k^{\text {th }}$ command input.

$$
\begin{gathered}
B(\bar{z}, \bar{v} \mid \vec{v})=\frac{1}{N} \cdot\left(\begin{array}{l}
N \\
y_{k}
\end{array}\right)\left[F\left(y_{k}\right)\right]^{k-1}\left[1-F\left(y_{k}\right)\right]^{N-k} \cdot p\left[\left(\begin{array}{l}
N \\
y_{k}
\end{array}\right) P^{y_{k}} q^{N-y_{k}}\right]^{2} \frac{y_{k}}{N} \\
\frac{1}{N} \cdot\left(\begin{array}{l}
N \\
y_{k}
\end{array}\right)\left[F\left(y_{k}\right)\right]^{k-1}\left[1-F\left(y_{k}\right)\right]^{N-k} \cdot p\left[\left(\begin{array}{l}
N \\
y_{k}
\end{array}\right) P^{y_{k}} q^{N-y_{k}}\right]^{2} \cdot \frac{y_{k}}{N}, \\
p \equiv f\left(y_{k}\right), a<y_{k}<b
\end{gathered}
$$

$B(\bar{z}, \bar{v} \mid \vec{v})$ of interacting $I^{\text {th }}$ and $f^{\text {th }}$ command input.

$$
\begin{aligned}
B(\bar{z}, \bar{v} \mid \vec{v})= & \frac{N !}{(i-1) !(j-i-1) !(N-j) !}\left[F\left(y_{i}\right)\right]^{i-1}\left[F\left(y_{j}\right)-F\left(y_{i}\right)\right]^{j-1} \\
& \cdot\left[1-F\left(y_{j}\right)\right]^{N-j} f\left(y_{i}\right) f\left(y_{j}\right) \cdot\left(\begin{array}{c}
N \\
y_{i}
\end{array}\right) P^{y_{i}} q^{N-y_{i}}\left(\begin{array}{c}
N-y_{i} \\
y_{j}-1
\end{array}\right) P^{y_{j}} q^{N-y_{j}-y_{i}} \\
= & \frac{N !}{(i-1) !(j-i-1) !(N-j) !}\left[F\left(y_{i}\right)\right]^{i-1}\left[F\left(y_{j}\right)-F\left(y_{i}\right)\right]^{j-i-1} \\
& \cdot\left[1-F\left(y_{j}\right)\right]^{N-j} p_{i} p_{j}\left[\left(\begin{array}{l}
N \\
y_{i}
\end{array}\right) p_{i}^{y_{i}} q_{i}^{N-y_{i}}\right]^{2} \cdot \frac{y_{i}}{N} \\
& \cdot\left[\left(\begin{array}{c}
N-y_{i} \\
y_{j}-1
\end{array}\right) p_{i}^{y_{i}} q_{i}^{N-y_{j}-y_{i}}\right]^{2} \cdot \frac{y_{j}}{N-y_{i}}
\end{aligned}
$$




$$
p_{i} \equiv f\left(y_{i}\right), p_{j} \equiv f\left(y_{j}\right), a<y_{i}<y_{j}<b
$$

\subsection{Optimal Probabilities}

$\frac{\mathrm{d}}{\mathrm{d} p} B\left(\bar{z}, \bar{v} \mid \vec{v}^{\prime}\right)=0$ to get optimal prob of command inputs. These derivatives yield:

$$
\begin{gathered}
(N-1)\left[1-F\left(y_{1}\right)\right]^{N-2}(-p)(p)\left[\left(\begin{array}{l}
N \\
y_{i}
\end{array}\right) p^{y_{1}} q^{N-y_{1}}\right]^{2} \frac{y_{1}}{N} \\
+\left[1-F\left(y_{1}\right)\right]^{N-1} \cdot\left[\left(\begin{array}{l}
N \\
y_{i}
\end{array}\right) p^{y_{1}} q^{N-y_{1}}\right]^{2} \frac{y_{1}}{N}+2 p\left[1-F\left(y_{1}\right)\right]^{N-1} \\
\cdot \frac{y_{1}}{N}\left[\left(\begin{array}{c}
N \\
y_{i}
\end{array}\right) p^{y_{1}} q^{N-y_{1}}\right]\left[\left(\begin{array}{l}
N \\
y_{1}
\end{array}\right) y_{1} p^{y_{1}-1} q^{N-y_{1}}-\left(N-y_{1}\right) p^{y_{1}} q^{N-y_{1}-1}\right]=0 \\
-\frac{p^{2}(N-1)}{1-F\left(y_{1}\right)} \frac{y_{1}}{N}+\frac{y_{1}}{N}+2 p\left[\left(\begin{array}{l}
N \\
y_{1}
\end{array}\right) p^{y_{1}} q^{N-y_{1}}\right]^{-1} \\
\cdot\left[\left(\begin{array}{l}
N \\
y_{i}
\end{array}\right) y_{1} p^{y_{1}-1} q^{N-y_{1}}-\left(N-y_{1}\right) p^{y_{1}} q^{N-y_{1}-1}\right] \frac{y_{1}}{N}=0 \\
1-\frac{p^{2}(N-1)}{1-F\left(y_{1}\right)}+2 p\left[\left(\begin{array}{l}
N \\
y_{1}
\end{array}\right) p^{y_{1}} q^{N-y_{1}}\right]^{-1} \\
\cdot\left[\left(\begin{array}{l}
N \\
y_{i}
\end{array}\right) y_{1} p^{y_{1}-1} q^{N-y_{1}}-\left(N-y_{1}\right) p^{y_{1}} q^{N-y_{1}-1}\right]=0
\end{gathered}
$$

Simplifying Equation (18) to get

$$
\begin{gathered}
1-\frac{p^{2}(N-1)}{1-F\left(y_{1}\right)}+2 p\left(\frac{y_{1}}{p}-\frac{N-y_{1}}{q}\right)=0 \\
1+2 p\left(\frac{y_{1}}{p}-\frac{N-y_{1}}{q}\right)=\frac{p^{2}(N-1)}{1-F\left(y_{1}\right)} \\
1+\frac{2 p}{p q}\left(q y_{1}-p\left(N-y_{1}\right)\right)=\frac{p^{2}(N-1)}{1-F\left(y_{1}\right)} \\
q+2\left(q y_{1}-p\left(N-y_{1}\right)\right)=\frac{p^{2} q(N-1)}{1-F\left(y_{1}\right)}=q+2\left(y_{1}(q+p)-N p\right) \\
1-F\left(y_{1}\right)=\frac{p^{2} q(N-1)}{q+2\left(y_{1}(q+p)-N p\right)} \\
\therefore F\left(y_{1}\right)=1-\frac{(N-1) p^{2} q}{q+2\left(y_{1}(q+p)-N p\right)}=1-\frac{(N-1) p^{2} q}{q+2\left(y_{1}-N p\right)}
\end{gathered}
$$

Similarly

$$
F\left(y_{N}\right)=1-\frac{(N-1) p^{2} q}{q+2\left(y_{N}(q+p)-N p\right)}=1-\frac{(N-1) p^{2} q}{q+2\left(y_{N}-N p\right)}
$$


$B\left(\bar{z}, \bar{v} / v^{\prime}\right)$ for intermediate $k^{\text {th }}$ command inputs

$$
\begin{aligned}
& \quad B\left(\bar{z}, \bar{v} / v^{\prime}\right)=\frac{1}{N}\left(\begin{array}{l}
N \\
y_{i}
\end{array}\right)\left[F\left(y_{k}\right)\right]^{k-1}\left[1-F\left(y_{k}\right)\right]^{N-k} p\left[\left(\begin{array}{l}
N \\
y_{i}
\end{array}\right) p^{y_{k}} q^{N-y_{k}}\right]^{2} \frac{y_{k}}{N} \\
& \frac{\mathrm{d}}{\mathrm{d} p} B\left(\bar{z}, \bar{v} / v^{\prime}\right)=0 \\
& \frac{\mathrm{d}}{\mathrm{d} p} B\left(\bar{z}, \bar{v} / v^{\prime}\right) \\
& =\frac{y_{k}}{N}\left(\begin{array}{l}
N \\
y_{i}
\end{array}\right)\left\{(k-1)\left[F\left(y_{k}\right)\right]^{k-2}(p) p\left[1-F\left(y_{k}\right)\right]^{N-k}\left[\left(\begin{array}{l}
N \\
y_{k}
\end{array}\right) p^{y_{k}} q^{N-y_{k}}\right]^{2}\right. \\
& +(N-k)\left[F\left(y_{k}\right)\right]^{k-1}\left[1-F\left(y_{k}\right)\right]^{N-k-1}(-p) p\left[\left(\begin{array}{l}
N \\
y_{i}
\end{array}\right) p^{y_{k}} q^{N-y_{k}}\right]^{2} \\
& +2 p\left[F\left(y_{k}\right)\right]^{k-1}\left[1-F\left(y_{k}\right)\right]^{N-k}\left[\left(\begin{array}{l}
N \\
y_{i}
\end{array}\right) y_{k} p^{y_{k}-1} q^{N-k}-\left(N-y_{k}\right) p^{y_{k}} q^{N-y_{k}-1}\right] \\
& \left.+\left[\left(\begin{array}{l}
N \\
y_{i}
\end{array}\right) p^{y_{k}} q^{N-y_{k}}\right]\right\}=0
\end{aligned}
$$

Simplifying Equation (27) to get

$$
\begin{aligned}
& 1+\frac{(k-1) p^{2}}{F\left(y_{k}\right)}-\frac{(N-k) p^{2}}{1-F\left(y_{k}\right)}+2 p\left(\frac{y_{k}}{p}-\frac{N-y_{k}}{q}\right)=0 \\
& \frac{F\left(y_{k}\right)\left[1-F\left(y_{k}\right)\right]+(k-1)\left[1-F\left(y_{k}\right)\right] p^{2}-(N-k) F\left(y_{k}\right) p^{2}}{F\left(y_{k}\right)\left[1-F\left(y_{k}\right)\right]} \\
& +\frac{2}{q}\left(q y_{k}-\left(N-y_{k}\right) p\right)=0 \\
& F\left(y_{k}\right)\left[1-F\left(y_{k}\right)\right]+p^{2}(k-1)\left[1-F\left(y_{k}\right)\right]-p^{2}-(N-k) F\left(y_{k}\right) \\
& =-\frac{2}{q}\left(y_{k}(q+p)-N p\right) F\left(y_{k}\right)\left[1-F\left(y_{k}\right)\right] \\
& F\left(y_{k}\right)-\left[F\left(y_{k}\right)\right]^{2}+p^{2}(k-1)-p^{2}(k-1) F\left(y_{k}\right)+p^{2} F\left(y_{k}\right)-p^{2}(N-k) F\left(y_{k}\right) \\
& =-\frac{2}{q}\left[y_{k}(q+p)-N p\right] F\left(y_{k}\right)+\frac{2}{q}\left[y_{k}(q+p)-N p\right]\left[F\left(y_{k}\right)\right]^{2} \\
& F\left(y_{k}\right)-\left[F\left(y_{k}\right)\right]^{2}+p^{2}(k-1)-k p^{2} F\left(y_{k}\right)+p^{2} F\left(y_{k}\right)-N p^{2} F\left(y_{k}\right)+k p^{2} F\left(y_{k}\right) \\
& =-\frac{2}{q}\left[y_{k}(q+p)-N p\right] F\left(y_{k}\right)-\frac{2}{q}\left[y_{k}(q+p)-N p\right]\left[F\left(y_{k}\right)\right]^{2} \\
& \left\{1-\frac{2}{q}\left[y_{k}(q+p)-N p\right]\right\}\left[F\left(y_{k}\right)\right]^{2} \\
& +\left\{-1+p^{2}(k-1)+p^{2}(N-k)-\frac{2}{q}\left[y_{k}(q+p)-N p\right]\right\} F\left(y_{k}\right)-p^{2}(k-1)=0 \\
& \left\{1-\frac{2}{q}\left[y_{k}(q+p)-N p\right]\right\}\left[F\left(y_{k}\right)\right]^{2} \\
& +\left\{(N-1) p^{2}-1-\frac{2}{q}\left[y_{k}(q+p)-N p\right]\right\} F\left(y_{k}\right)-p^{2}(k-1)=0
\end{aligned}
$$


By quadratic equation: $a x^{2}+b x+c=0$, solution: $(x-\alpha)(x-\beta)=0$, $(\alpha, \beta)=\frac{-b \pm \sqrt{b^{2}-4 a c}}{2 a}$

Let

$$
\begin{gathered}
F\left(y_{i}\right)=x \\
\therefore F\left(y_{k}\right)=\frac{1-(N-1) p^{2}+\frac{2}{q}\left[y_{k}(q+p)-N p\right]}{2\left\{1-\frac{2}{q}\left[y_{k}(q+p)-N p\right]\right\}} \\
\pm \frac{\sqrt{\left\{1-(N-1) p^{2}+\frac{2}{q}\left[y_{k}(q+p)-N p\right]\right\}+4\left\{1-\frac{2}{q}\left[y_{k}(q+p)-N p\right](k-1) p^{2}\right\}}}{2\left\{1-\frac{2}{q}\left[y_{k}(q+p)-N p\right]\right\}} \\
F\left(y_{i j}\right)=\frac{N !}{(i-1) !(j-i-1) !(N-j) !}\left[F\left(y_{i}\right)\right]^{i-1} p_{i}\left[\left(\begin{array}{l}
N \\
y_{i}
\end{array}\right) p_{i}^{y_{i}} q^{N-y_{i}}\right]^{2} \frac{y_{i}}{N} \\
\cdot\left[F\left(y_{j}\right)-F\left(y_{i}\right)\right]^{j-i-1}\left[1-F\left(y_{j}\right)\right]^{N-j} p_{j}\left[\left(\begin{array}{l}
N \\
y_{j}
\end{array}\right) p_{j}^{y_{j}} q^{N-y_{j}-y_{i}}\right]^{2} \frac{y_{j}}{N-y_{i}}
\end{gathered}
$$

Let

$$
\begin{gathered}
\phi_{1}=\left[F\left(y_{i}\right)\right]^{i-1} p_{i}\left[\left(\begin{array}{l}
N \\
y_{i}
\end{array}\right) p_{i}^{y_{i}} q_{i}^{N-y_{i}}\right]^{2} \frac{y_{i}}{N} \\
\phi_{2}=\left[F\left(y_{j}\right)-F\left(y_{i}\right)\right]^{j-i-1} \\
\phi_{3}=\left[1-F\left(y_{j}\right)\right]^{N-j} p_{j}\left[\left(\begin{array}{c}
N-y_{i} \\
y_{j}-1
\end{array}\right) p_{j}^{y_{j}} q_{j}^{N-y_{j}-y_{i}}\right]^{2} \frac{y_{j}}{N-y_{i}} \\
\frac{\mathrm{d}}{\mathrm{d} p} B\left(\bar{z}, \bar{v} / v^{\prime}\right)=\phi_{1}^{\prime} \phi_{2} \phi_{3}+\phi_{1} \phi_{2}^{\prime} \phi_{3}+\phi_{1} \phi_{2} \phi_{3}^{\prime}=0 \\
\phi_{1}^{\prime}=(i-1)\left[F\left(y_{i}\right)\right]^{i-2} p_{i}^{2}\left[\left(\begin{array}{l}
N \\
y_{i}
\end{array}\right) p_{i}^{y_{i}} q^{N-y_{i}}\right]^{2}+\left[F\left(y_{i}\right)\right]^{i-1}\left[\left(\begin{array}{l}
N \\
y_{i}
\end{array}\right) p_{i}^{y_{i}} q^{N-y_{i}}\right]^{2} \\
+2 p_{i}\left[F\left(y_{i}\right)\right]^{i-1}\left[\left(\begin{array}{l}
N \\
y_{i}
\end{array}\right) p^{y_{i}} q^{N-y_{i}}\right]\left[\left(N-y_{i}\right) p^{y_{i}} q^{N-y_{i}-1}\right] \frac{y_{i}}{N} \\
y_{i} \cdot\left\{\frac{(i-1) p_{i}^{2}}{F\left(y_{i}\right)}+1+2 p_{i}\left[\frac{y_{i}}{p_{i}}-\frac{\left(N-y_{i}\right)}{q_{i}}\right]\right\}\left[F\left(y_{i}\right)\right]^{i-1}\left[\left(\begin{array}{l}
N \\
y_{i}
\end{array}\right) p_{i}^{y_{i}} q_{i}^{N-y_{i}}\right]^{2} \frac{y_{i}}{N} \\
\left\{(i-1)\left[F\left(y_{i}\right)\right]^{-1} p_{i}^{2}+1+\frac{2}{q_{i}}\left[y_{i}\left(q_{i}+p\right)-N p_{i}\right]\right\}\left[F\left(y_{i}\right)\right]^{i-1}\left[\left(\begin{array}{l}
N \\
y_{i}
\end{array}\right) p_{i}^{y_{i}} q^{N-y_{i}}\right]^{2} \\
(j-i-1)\left[F\left(y_{j}\right)-F\left(y_{i}\right)\right]^{j-i-2}\left(p_{j}-p_{i}\right)
\end{gathered}
$$




$$
\begin{aligned}
& \phi_{3}^{\prime}=(N-j)\left[1-F\left(y_{i}\right)\right]^{N-j-1}\left(-p_{j}\right) p_{j}\left[\left(\begin{array}{c}
N-y_{i} \\
y_{j}-1
\end{array}\right) p_{i}^{y_{i}} q_{j}^{N-y_{j}-y_{i}}\right]^{2} \\
& +\left[1-F\left(y_{j}\right)\right]^{N-j}\left[\left(\begin{array}{c}
N-y_{i} \\
y_{j}-1
\end{array}\right) p_{j}^{y_{j}} q_{j}^{N-y_{j}-y_{i}}\right]^{2} \\
& +2\left[1-F\left(y_{j}\right)\right]^{N-j} p_{j}\left[\left(\begin{array}{c}
N-y_{i} \\
y_{j}-1
\end{array}\right) p_{j}^{y_{j}} q_{j}^{N-y_{j}-y_{i}}\right] \\
& \cdot\left[\left(\begin{array}{c}
N-y_{i} \\
y_{j}-1
\end{array}\right)\left(y_{j} p_{j}^{y_{j}} q_{j}^{N-y_{j}-y_{i}}-\left(N-y_{j}-y_{i}\right) p_{j}^{y_{j}} q_{j}^{N-y_{j}-y_{i}}\right)\right] \cdot \frac{y_{j}}{N-y_{i}} \\
& =\left\{-\frac{(N-j) p_{j}^{2}}{1-F\left(y_{j}\right)}+1+2 p_{j}\left[\frac{y_{j}}{p_{j}}-\frac{N-y_{j}-y_{i}}{q_{j}}\right]\right\} \\
& \cdot\left[1-F\left(y_{j}\right)\right]^{N-j}\left[\left(\begin{array}{c}
N-y_{i} \\
y_{j}-1
\end{array}\right) p_{j}^{y_{j}} q_{j}^{N-y_{j}-y_{i}}\right]^{2} \cdot \frac{y_{j}}{N-y_{i}} \\
& =\frac{y_{j}}{N-y_{i}}\left\{-(N-j) p_{j}^{2}\left[1-F\left(y_{j}\right)\right]^{-1}+1+\frac{2}{q_{j}}\left[y_{j}\left(q_{j}+p_{j}\right)-N p_{j}\right]\right\} \\
& \cdot\left[1-F\left(y_{j}\right)\right]^{N-j}\left[\left(\begin{array}{c}
N-y_{i} \\
y_{j}-1
\end{array}\right) p_{j}^{y_{j}} q_{j}^{N-y_{j}-y_{i}}\right]^{2} \\
& \frac{\mathrm{d} B}{\mathrm{~d} p}\left(\bar{z}, \bar{v} \backslash v^{\prime}\right) \\
& =\left\{\frac{(i-1) p_{i}^{2}}{F\left(y_{i}\right)}+1+\frac{2}{p_{j}}\left[y_{j}\left(q_{i}+p_{i}\right)-N p_{i}\right]\right\} B\left(\bar{z}, \bar{v} / v^{\prime}\right) \\
& +(j-i-1)\left(p_{j}-p_{i}\right)\left[F\left(y_{j}\right)-F\left(y_{i}\right)\right]^{-1} B\left(\bar{z}, \bar{v} / v^{\prime}\right) \\
& +\left\{-(N-j) p_{j}^{2}\left[1-F\left(y_{j}\right)\right]^{-1}+1-\frac{2}{q_{j}}\left[y_{j}\left(q_{j}+p_{j}\right)-N p_{j}\right]\right\} B\left(\bar{z}, \bar{v} / v^{\prime}\right) \\
& =(i-1) p_{i}^{2}\left[F\left(y_{i}\right)\right]^{-1}+1-\frac{2}{q_{i}}\left[y_{i}\left(q_{i}+p_{i}\right)-N p_{i}\right] \\
& +(j-i-1)\left(p_{j}+p_{i}\right)\left[F\left(y_{j}\right)-F\left(y_{i}\right)\right]^{-1} \\
& -(N-j) p_{j}^{2}\left[1-F\left(y_{j}\right)\right]^{-1}+1-\frac{2}{q_{j}}\left[y_{j}\left(q_{j}+p_{j}\right)-N p_{j}\right] \\
& =0
\end{aligned}
$$

\section{Simplifying Equation (44) to get}

$$
\begin{aligned}
& (i-1) p_{i}^{2}\left[F\left(y_{j}\right)-F\left(y_{i}\right)\right]\left[1-F\left(y_{i}\right)\right] \\
& +\left\{1+\frac{2}{q_{i}}\left[y_{i}\left(q_{i}+p_{i}\right)-N p_{i}\right]\right\} F\left(y_{i}\right)\left[F\left(y_{j}\right)-F\left(y_{i}\right)\right]\left[1-F\left(y_{j}\right)\right] \\
& +(j-i-1)\left(p_{j}-p_{i}\right) F\left(y_{i}\right)\left[1-F\left(y_{j}\right)\right]-(N-j) p_{j}^{2} F\left(y_{i}\right)\left[F\left(y_{j}\right)-F\left(y_{i}\right)\right] \\
& +\left\{1-\frac{2}{q_{j}}\left[y_{j}\left(q_{j}+p_{j}\right)-N p_{j}\right]\right\}\left[F\left(y_{i}\right)-F\left(y_{j}\right)-F\left(y_{i}\right)\right]=0
\end{aligned}
$$




$$
\begin{aligned}
& \quad(i-1) p_{i}^{2}\left[F\left(y_{j}\right)-F\left(y_{j}\right)^{2}-F\left(y_{i}\right)+F\left(y_{i}\right) F\left(y_{j}\right)\right] \\
& +\left\{1-\frac{2}{q_{i}}\left[y_{i}\left(q_{i}+p_{i}\right)-N p_{i}\right]\right\} F\left(y_{i}\right)\left[F\left(y_{j}\right)-F\left(y_{i}\right) F\left(y_{j}\right)^{2}\right]\left[1-F\left(y_{j}\right)\right] \\
& +(j-i-1)\left(p_{j}-p_{i}\right) F\left(y_{i}\right)\left[1-F\left(y_{j}\right)\right]-(N-j) p_{j}^{2} F\left(y_{i}\right)\left[F\left(y_{j}\right)-F\left(y_{i}\right)\right](46) \\
& \quad+\left\{1-\frac{2}{q_{j}}\left[y_{j}\left(q_{j}+p_{j}\right)-N p_{j}\right]\right\}\left[F\left(y_{i}\right)-F\left(y_{j}\right)-F\left(y_{i}\right) F\left(y_{j}\right)^{2}\right] \\
& \quad \cdot\left[F\left(y_{i}\right)^{2}+F\left(y_{j}\right) F\left(y_{i}\right)^{2}\right]=0 \\
& \left\{(N-j) p_{j}^{2}-1+\frac{2}{q_{j}}\left[y_{j}\left(q_{j}+p_{j}\right)-N p_{j}\right]-1-\frac{2}{q_{j}}\left[y_{i}\left(q_{i}+p_{i}\right)-N p_{i}\right]\right\}\left(F\left(y_{i}\right)\right)^{2} \\
& +\left[(j-i-1)\left(p_{j}-p_{i}\right)-(i-1) p_{i}^{2}\right] F\left(y_{i}\right)-\left[(i-1) p_{i}^{2}\left(F\left(y_{j}\right)\right)^{2}-F\left(y_{i}\right)\right] \\
& +\left[(i-1) p_{i}^{2}\right]\left(F\left(y_{j}\right)\right)^{2}-F\left(y_{j}\right)+\left\{1+\frac{2}{q_{i}}\left[y_{i}\left(q_{i}+p_{i}\right)-N p_{i}\right]\right\} \\
& \cdot\left[F\left(y_{j}\right)\left(F\left(y_{i}\right)\right)^{2}-\left[F\left(y_{i}\right)\left(F\left(y_{j}\right)\right)^{2}\right]+\left\{(i-1) p_{i}^{2}+1+\frac{2}{q_{i}}\left[y_{i}\left(q_{i}+p_{i}\right)-N p_{i}\right]\right.\right. \\
& \left.-\left[(j-i-1)\left(p_{j}-p_{i}\right)-(N-j) p_{j}^{2}-1-\frac{2}{q_{j}}\left[y_{j}\left(q_{j}+p_{j}\right)-N p_{j}\right]\right\} F\left(y_{i}\right) F\left(y_{j}\right)\right]=0
\end{aligned}
$$

Then from Equation (35)

$$
\begin{gathered}
\left\{(N-j) p_{j}^{2}-1+\frac{2}{q_{j}}\left[y_{j}\left(q_{j}+p_{j}\right)-N p_{j}\right]-1-\frac{2}{q_{i}}\left[y_{i}\left(q_{i}+p_{i}\right)-N p_{i}\right]\right\}\left(F\left(y_{i}\right)\right)^{2} \\
+\left[(j-i-1)\left(p_{j}-p_{i}\right)-(i-1) p_{i}^{2}\right] F\left(y_{i}\right)+1=0 \\
\therefore F\left(y_{i}\right)=\frac{-(j-i-1)\left(p_{j}-p_{i}\right)-(i-1) p_{i}^{2} \pm \sqrt{\left[(j-i-1)\left(p_{j}-p_{i}\right)-(i-1) p_{i}^{2}\right]^{2}-4\left\{(N-j) p_{j}^{2}-1\right\}}}{2\left\{(N-j) p_{j}^{2}-1+\frac{2}{q_{j}}\left[y_{j}\left(q_{j}+p_{j}\right)-N p_{j}\right]-1-\frac{2}{q_{i}}\left[y_{i}\left(q_{i}+p_{i}\right)-N p_{i}\right]\right\}} \\
+\frac{\frac{2}{q_{j}}\left[y_{j}\left(q_{j}+p_{j}\right)-N p_{j}\right]-1-\frac{2}{q_{i}}\left[y_{i}\left(q_{i}+p_{i}\right)-N p_{i}\right]\left[(i-1) p_{i}^{2}\right]\left[\left(F\left(y_{j}\right)\right)^{2}-F\left(y_{j}\right)\right]-1}{2\left\{(N-j) p_{j}^{2}-1+\frac{2}{q_{j}}\left[y_{j}\left(q_{j}+p_{j}\right)-N p_{j}\right]-1-\frac{2}{q_{i}}\left[y_{i}\left(q_{i}+p_{i}\right)-N p_{i}\right]\right\}}=0
\end{gathered}
$$

Simplifying the above quadratic equations to get

$$
\begin{gathered}
-\left[(i-1) p_{i}^{2}\right]\left[\left(F\left(y_{j}\right)\right)^{2}-F\left(y_{j}\right)\right]-1=0 \\
\Rightarrow(i-1) p_{i}^{2}\left[\left(F\left(y_{j}\right)\right)^{2}-F\left(y_{j}\right)\right]+1=0 \\
\therefore F\left(y_{j}\right)=\frac{(i-1) p_{i}^{2} \pm \sqrt{(i-1) p_{i}^{2}-4(i-1) p_{i}^{2}}}{2(i-1) p_{i}^{2}}
\end{gathered}
$$


But

$$
F\left(y_{j}\right)\left(F\left(y_{i}\right)\right)^{2}-F\left(y_{i}\right)\left(F\left(y_{j}\right)\right)^{2}=F\left(y_{i}\right) F\left(y_{j}\right)\left[F\left(y_{i}\right)-F\left(y_{j}\right)\right]
$$

Then

$$
\begin{gathered}
\left\{1+\frac{2}{q_{i}}\left[y_{i}\left(q_{i}+p_{i}\right)-N p_{i}\right]\right\}\left[F\left(y_{i}\right)-F\left(y_{j}\right)\right] \\
=-(i-1) p_{i}^{2}+\frac{2}{q_{i}}\left[y_{i}\left(q_{i}+p_{i}\right)-N p_{i}\right]-(j-i-1)\left(p_{j}-p_{i}\right) \\
-(N-j) p_{j}^{2}-1-\frac{2}{q_{j}}\left[y_{j}\left(q_{j}+p_{j}\right)-N p_{j}\right] \\
\therefore F\left(y_{j}\right)-F\left(y_{i}\right)=\frac{(i-1) p_{i}^{2}+1+\frac{2}{q_{i}}\left[y_{i}\left(q_{i}+p_{i}\right)-N p_{i}\right]-(j-i-1)\left(p_{j}-p_{i}\right)}{1+\frac{2}{q_{i}}\left[y_{i}\left(q_{i}+p_{i}\right)-N p_{i}\right]} \\
+\frac{1+\frac{2}{q_{i}}\left[y_{i}\left(q_{i}+p_{i}\right)-N p_{i}\right]}{N-j) p_{j}^{2}-1-\frac{2}{q_{j}}\left[y_{j}\left(q_{j}+p_{j}\right)-N p_{j}\right]} \\
=1+\frac{(i-1) p_{i}^{2}-(j-i-1)\left(p_{j}-p_{i}\right)-(N-j) p_{j}^{2}-1-\frac{2}{q_{j}}\left[y_{j}\left(q_{j}+p_{j}\right)-N p_{j}\right]}{\left.\left.1+p_{i}\right)-N p_{i}\right]}
\end{gathered}
$$

\section{Results}

$$
\begin{aligned}
& F\left(y_{1}\right)=1-\frac{(10-1)(0.3)^{2}(0.7)}{0.7+2(1-3)}=1-\frac{0.567}{-3.3}=1+0.171818=1.172 \text { unrealistic } \\
& F\left(y_{3}\right)=1-\frac{9(0.3)^{2}(0.7)}{0.7}=1-0.81=0.19 \\
& F\left(y_{10}\right)=1-\frac{(10-1)(0.3)^{2}(0.7)}{0.7+2(10-3)}=1-\frac{0.567}{14.7}=1-0.0386=0.9614
\end{aligned}
$$

These results indicate:

1) $F\left(y_{1}\right)$ not feasible due to no (or low) command input(s).

2) $F\left(y_{3}\right)$ feasible.

3) $F\left(y_{10}\right)$ feasible. As the hierarchy increases correspondingly the distribution increases. This can be taken as workload.

Example 1: Given $p_{k}=0.3, k=5, N=10, y_{k}=k$

$$
\begin{aligned}
& F\left(y_{k}\right)=F\left(y_{5}\right)=\frac{1-9(0.09)+\frac{2}{0.7}(5-10(0.3))}{2\left\{1+\frac{2}{0.7}(5-10(0.3)\}\right.} \\
& \pm \frac{\sqrt{\left\{-1+9(0.09)-\frac{2}{0.7}(5-10(0.3)\}^{2}+4\left(1+\frac{2}{0.7}(5-10(0.3)) 4(0.09)\right)\right.}}{2\left\{1+\frac{2}{0.7}(5-10(0.3)\}\right.}
\end{aligned}
$$




$$
\begin{aligned}
& =\frac{1-0.81+\frac{4}{0.7} \pm \sqrt{\left(-0.19+\frac{4}{0.7}\right)^{2}+4\left(1+\frac{4}{0.7}(0.36)\right)}}{2\left\{1+\frac{4}{0.7}\right\}} \\
& =\frac{0.12+5.7143 \pm \sqrt{(5.5243)^{2}+4(3.0572)}}{2(6.7143)} \\
& =\frac{5.8343 \pm \sqrt{30.5179+12.288}}{13.4286}=\frac{5.8343 \pm \sqrt{42.7467}}{13.4286}=\frac{5.8343 \pm 6.5381}{13.4286} \\
& \frac{12.3724}{13.4286},-\frac{0.7467}{13.4286}=0.92135,-0.05241(\text { unrealistic })
\end{aligned}
$$

Example 2: Given $p_{i}=0.3, p_{j}=0.5, i=4, j=7, k=5, N=10, y_{k}=K$

$$
\begin{aligned}
F & \left(y_{4}\right)=F\left(y_{4}\right)=\frac{-(2(0.2)-3(0.09))}{2\left\{3(0.25)-1+\frac{2}{0.5}[7-5]-1-\frac{2}{0.7}[4-3]\right\}} \\
& \pm \frac{\sqrt{[2(0.2)-3(0.09)]^{2}+4\left\{3(0.25)-1+\frac{2}{0.5}[7-5]-1-\frac{2}{0.7}[4-3]\right\}}}{2\left\{3(0.25)-1+\frac{2}{0.5}[7-5]-1-\frac{2}{0.7}[4-3]\right\}} \\
= & \frac{-(0.4-0.27) \pm \sqrt{(0.4-0.27)^{2}+4\{0.75-1+4(2)-1-2.8571\}}}{2\{0.75-1+4(2)-1-2.8571\}} \\
= & \frac{-0.13 \pm \sqrt{15.5885}}{7.7858}=\frac{-0.13 \pm 3.9482}{7.7858} \\
= & \frac{3.8182}{7.7858}=0.49041, \frac{-4.0782}{7.7858}(\text { unrealistic }) \\
& F\left(y_{j}\right)=F\left(y_{7}\right)=\frac{3(0.09) \pm \sqrt{(3(0.09))^{2}+4[3(0.09)]}}{2(3(0.09)} \\
& =\frac{0.27 \pm \sqrt{0.0729+1.08}}{0.54}=\frac{0.27 \pm \sqrt{0.1529}}{0.54}=\frac{0.27 \pm 1.073732}{0.54} \\
& =\frac{1.343732}{0.54}=2.4884, \frac{-0.803732}{0.54}=-1.4884(\text { both unrealistic }) \\
\approx & 0 \text { certainly unrealistic }
\end{aligned}
$$$$
F\left(y_{j}\right)=F\left(y_{i}\right)=\frac{3(0.09)+1+\frac{2}{0.7}[4-3]-2(0.2)-3(0.25)-1-\frac{2}{0.5}[7-5]}{1+\frac{2}{0.7}-2(0.2)-1-\frac{2}{0.5}(2)}
$$$$
=\frac{-6.0229}{-5.5429}=1.0866
$$

$\approx 0$ certainly unrealistic

\section{Discussion}

The distribution of events at various stages of interactions were convolutions to obtain system distribution. Thereafter, analytical approach was used at various 
stages to obtain optimal distribution of the command inputs.

\section{Conclusion}

Optimal distribution values obtained can be taken as system efficiency of the model, which can be used for system control.

\section{Conflicts of Interest}

The authors declare no conflicts of interest regarding the publication of this paper.

\section{References}

[1] Boettcher, K.L. and Levis, A.H. (1982) Modelling the Interacting Decision Maker with Bounded Rationality. IEEE Transactions on Systems, Man, and Cybernetics, 12, 334-344. https://doi.org/10.1109/TSMC.1982.4308823

[2] Oladejo, M.O. (2013) Modelling N-Team of Interacting Decision Makers with Bounded Rationality Constraints. Medwell Journal. (Accepted and Processing Fee Paid but Yet to Receive Hard Copy)

[3] Oladejo, M.O. (2014) Spectral Analysis of Modelled N-Team Interacting Decision Makers with Bounded Rationality constraints. Asian Journal and Applied Sciences, 3, 20-48.

[4] Oladejo, M.O. and Aligho, C.M. (2013) Optimal Queuing Modell of Medical Centres: A Case Study of Shehu Mohammed Kangiwa Medical Centre, Kaduna Polytechnic. IOSR, Journal of Mathematics, 10, 18-22. https://doi.org/10.9790/5728-10111822

[5] Okafor, U.L. and Oladejo, M.O. (2014) Modelling Cadets Offences by Interaction Effects. PJA/PJER, 2, 89-95.

[6] Oladejo, M.O., Agashua, N.U. and Jighijighi, A.T. (2015) Optimizing the Queuing System (Model) of a Fast Food Restaurant: A case study of Ostrich Bakery. International Journal of Recent Development in Engineering \& Technology, 4, 1-10.

[7] Oladejo, M.O. and Agashua, N.U. (2015) Optimizing the Product Mixings and Machine Processing Time of a Fast Food Restaurant Using a Linear Programming Model: Case Study of Ostrich Bakery. Asian Journal of Science and Technology, 6, 2043-2047.

[8] Oladejo, M.O. and Jighjigh, A.T. (2014) Optimal Transportation Network Using Challenges as Catalytic Factors on Insurgent Activities Characterize Route to Maiduguri. AJST, 5, 448-474.

[9] Oladejo, M.O. and Amoka, J.A. (2015) Optimal Replacement Policy for Mass Transit Vehicle Operation KSTA: A Case Study.

[10] Ibrahim, S.U., Oladejo, M.O. and Okafor, U.L. (2018) Application of ARIMA and Difference Equation Models for Forecasting Hazards of some Climatic Factors in Sokoto Metropolis. Journal of the Nigerian Association of Mathematical Physics, 44, 177-184.

[11] Oladejo, M.O. and Bello, A. (2015) A Suitable Model for the Forecast of Exchange Rate in Nigeria (Nigerian Naira versus US Dollar. International Journal of Science and Research (IJSR), 4, 2669-2676.

[12] Oladejo, M.O. and Ali Onuche, J. (2016) A Model for the Assessment, Monitoring, Control, and Evaluation of HIV/AIDS Confirmed Status with Vertical Transmission 
in Nigeria. 32, 460-462.

[13] Oladejo, M.O. and Ali, J.O. (2017) A Steady State Solution Method for HIV/AIDS Model for the Assessment, Monitoring, Control and Evaluation of Confirmed Status with Vertical Transmission in Nigeria. Journal of Virology \& Antiviral Research, 6, 1-7. https://doi.org/10.4172/2324-8955.1000166 